\title{
Molecular characterization of a rice mutator-phenotype derived from an incompatible cross-pollination reveals transgenerational mobilization of multiple transposable elements and extensive epigenetic instability
}

\author{
Hongyan Wang ${ }^{\dagger 1}$, Yang Chai ${ }^{\dagger 1}$, Xiucheng $\mathrm{Chu}^{\dagger 2}$, Yunyang Zhao ${ }^{1}$, Ying $\mathrm{Wu}^{1}$, \\ Jihong Zhao ${ }^{2}$, Frédéric Ngezahayo ${ }^{1}$, Chunming $\mathrm{Xu}^{* 1}$ and Bao Liu*1,3
}

\begin{abstract}
Address: ${ }^{1}$ Key Laboratory of Molecular Epigenetics of MOE and Institute of Genetics \& Cytology, Northeast Normal University, Changchun 130024, PR China, ${ }^{2}$ Tonghua Academy of Agricultural Sciences, Hailong 135007, Jilin Province, PR China and ${ }^{3}$ Key Laboratory of Applied Statistics of MOE, Northeast Normal University, Changchun 130024, PR China

Email: Hongyan Wang - hongyan2003@126.com; Yang Chai - muzi0926@163.com; Xiucheng Chu - chuxiucheng@163.com;

Yunyang Zhao - zhaoyunyang2002@163.com; Ying Wu - wuy003@nenu.edu.cn; Jihong Zhao - jihongzhao@163.com;

Frédéric Ngezahayo -ngezafre@yahoo.fr; Chunming Xu* - xucm848@nenu.edu.cn; Bao Liu* - baoliu@nenu.edu.cn

* Corresponding authors †Equal contributors
\end{abstract}

Published: 29 May 2009

BMC Plant Biology 2009, 9:63 doi:10.1 I86/147I-2229-9-63

This article is available from: http://www.biomedcentral.com/I47I-2229/9/63

(c) 2009 Wang et al; licensee BioMed Central Ltd.

This is an Open Access article distributed under the terms of the Creative Commons Attribution License (http://creativecommons.org/licenses/by/2.0), which permits unrestricted use, distribution, and reproduction in any medium, provided the original work is properly cited.
Received: 16 January 2009

Accepted: 29 May 2009

which permits unrestricted use, distribution, and reproduction in any medium, provided the original work is properly cited.

\begin{abstract}
Background: Inter-specific hybridization occurs frequently in plants, which may induce genetic and epigenetic instabilities in the resultant hybrids, allopolyploids and introgressants. It remains unclear however whether pollination by alien pollens of an incompatible species may impose a "biological stress" even in the absence of genome-merger or genetic introgression, whereby genetic and/or epigenetic instability of the maternal recipient genome might be provoked.
\end{abstract}

Results: We report here the identification of a rice mutator-phenotype from a set of rice plants derived from a crossing experiment involving two remote and apparently incompatible species, Oryza sativa L. and Oenothera biennis L. The mutatorphenotype (named Tong2 I I-LP) showed distinct alteration in several traits, with the most striking being substantially enlarged panicles. Expectably, gel-blotting by total genomic DNA of the pollen-donor showed no evidence for introgression. Characterization of Tong2 I I-LP (SO) and its selfed progenies (SI) ruled out contamination (via seed or pollen) or polyploidy as a cause for its dramatic phenotypic changes, but revealed transgenerational mobilization of several previously characterized transposable elements (TEs), including a MITE (mPing), and three LTR retrotransposons (Osr7, Osr23 and Tos /7). AFLP and MSAP fingerprinting revealed extensive, transgenerational alterations in cytosine methylation and to a less extent also genetic variation in Tong2 I I-LP and its immediate progenies. mPing mobility was found to correlate with cytosine methylation alteration detected by MSAP but not with genetic variation detected by AFLP. Assay by q-RT-PCR of the steady-state transcript abundance of a set of genes encoding for the various putative DNA methyltransferases, 5-methylcytosine DNA glycosylases, and small interference RNA (siRNA) pathway-related proteins showed that, relative to the rice parental line, heritable perturbation in expression of 12 out of the 13 genes occurred in the mutator-phenotype and its sefled progenies.

Conclusion: Transgenerational epigenetic instability in the form of altered cytosine methylation and its associated TE activity occurred in a rice mutator-phenotype produced by pollinating the rice stigma with pollens of 0 . biennis. Heritably perturbed homeostatic expression-state of genes involved in maintenance of chromatin structure is likely an underlying cause for the alien pollination-induced transgenerational epigenetic/genetic instability, and which occurred apparently without entailing genome merger or genetic introgression. 


\section{Background}

It is widely accepted that hybridization between genetically differentiated natural plant populations is a frequent phenomenon, which contributes to genome evolution, and can lead to speciation via allopolyploidy or at the homoploid level [1-6]. Apart from the properties of hybridization that can be explained by classical genetic mechanisms such as direct transfer and/or recombinatory generation of beneficial alleles, recent studies in both plant and animals have revealed that wide hybridization may generate variations by novel means such as rapid structural genomic changes, novel gene expression trajectories and epigenetic alterations, which apparently transgress Mendelian principles [1,7-17] One possible mechanism for the occurrence of non-Mendelian genomic and transcriptomic changes as a result of hybridization is lato sensu the "genomic shock" hypothesis proposed by McClintock [18].

Several lines of circumstantial evidence have suggested that hybridization-associated genetic and epigenetic instabilities may also be provoked in unsuccessful or "abortive" hybridizations between distant and sexually incompatible species. For example, it was found that random integration of uncharacterized DNA segments from unrelated sources into cultured animal cells, and introgression of multiple, tiny chromatin segments from a distantly related donor species into a recipient plant species may be mutagenic and induce genetic and epigenetic variations [19-23]. Although in these instances, the introgression of alien DNA or chromatin segments were automatically assumed as the causal factor for the induced instabilities, no direct link between the two events was ever established. In fact, a common observation emerged from these studies has indicated that the genomic loci underwent the changes are largely random both with regard to their chromosomal distribution and to nature of the changed sequences, thus argues against localized effects (e.g., insertional mutagenesis). Therefore, it remained a formal possibility that at least some of the detected non-Mendelian genetic and epigenetic mutations in these cases may not have been induced by the integration of DNA or chromatin segments per se; instead, they might have been the consequence of the process of genetic transfer (in animals) or alien pollination (in plants), which conceivably may constitute a kind of "biological stress" and elicit genetic and epigenetic instabilities, a scenario consistent with McClintock's "genomic shock" hypothesis [18].

In theory, it is possible that the process of pollination by pollens even from a remote and incompatible species may constitute a "biological stress" to the recipient parent in myriad ways. For example, metabolites including small signal molecules (e.g., nitric oxide and reactive oxygen species [24]) and various phytohormones of the alien pollens may enter stigma cells of the recipient species during their physical contacts; conceivably, this may induce physiological and biochemical mismatches of various kinds. Consequently, if the cellular machinery responsible for the constant fine-tuning of chromatin structure is compromised, then the occurrence of epigenetic and even genetic instability is almost inevitable. In this regard, the pollination by alien pollens from an incompatible species may bear mechanistic resemblance to pathogen attack wherein the pathogen's DNA or RNA usually does not integrate into the host genome, yet its interaction with the host may cause genetic and epigenetic instabilities in the latter. Indeed, it was documented recently in tobacco that pathogen infestation caused both general genetic instability (due to increased somatic recombination) and alteration in cytosine methylation at specific loci in the infected plants, and both of which are heritable to successive biological generations $[25,26]$.

The aim of this study was to explore if pollination by alien pollens from an extremely remote and apparently incompatible plant species, which obviously would not generate genome merger or genetic introgressions, may still impose a "biological stress" to the recipient maternal genome, and induce heritable genetic and epigenetic instabilities.

\section{Results and discussion \\ Identification of a mutator-phenotype}

We harvested more than 300 seeds from ca. 50 rice (Oryza sativa L. ssp. japonica, cv. Tong211) panicles that were artificially pollinated by fresh pollens taken from a single accession of a dicot plant, evening primrose (Oenothera biennis L.), followed by a second round pollination $48 \mathrm{hrs}$ later with their own pollens collected from other individuals of Tong211 - a pollination method we termed "repeated pollination" [27]. We found that a substantial portion of the seeds showed abnormal development as a result of the manipulation, and which either did not germinate or died at early seedling stages. Among 84 rice plants (designated as S0 generation) growing to maturity, we identified a single individual plant (hereforth named Tong211-LP) that showed conspicuous phenotypic variation in multiple traits, including flowering time, seed-size, and particularly panicle-size (larger panicle, LP), relative to its rice parental cultivar. We then self-pollinated several panicles of this plant to produce seeds (also markedly enlarged), and the S1 progeny plants. Tong211-LP was partially sterile and produced a much smaller quantity of seeds than expected from a normal rice individual. Nonetheless, among the progenies produced, phenotypic variations that were observed in the individual mutatorphenotype (S0) were inherited at high frequencies to the $\mathrm{S} 1$ progenies; in addition, some new phenotypic variations that were apparently individual-specific appeared $d e$ 
novo in some of the S1 progeny plants. Typical phenotypic variations in the S1 progeny plants of the mutator-phenotype were shown in Figure 1. The transgenerational continuous occurrence of multiple phenotypic variations, i.e., sustained phenotypic instability, from a single individual is characteristic of a "mutator-phenotype", as originally reported in Drosophila [28].

\section{Transpositional activation of multiple TEs in the mutator-} phenotype and its selfed progenies

Genomic DNA was isolated from expanded young leaves of Tong211-LP (vegetatively propagated from root-stock cuttings), its eight selected S1 progeny individuals that exhibited the most extreme phenotypic variations, the parental rice line (cv. Tong211) and the pollen-donor, $O$. biennis. A series of DNA gel blot analysis was performed. First, to look for possible genetic introgression from the pollen donor $O$. bienni into rice, we did a genomic DNA probing assay [29], i.e., using labeled genomic DNA of $O$. biennis as a probe and autoclaved genomic DNA (150x excess in quantity) of rice as a blocker. We detected no evidence for genomic introgression (data not shown) based on this assay, as expected given the extreme phylogenetic remoteness of the two species. It should be pointed out however that this genomic blotting assay, though efficient [29], is by no means exclusive as only introgression of $O$. biennis species-specific genomic sequences would have been detected, and therefore cryptic introgression incidents may go undetected. Next, given the transgenerational mutability of multiple phenotypic traits, we asked

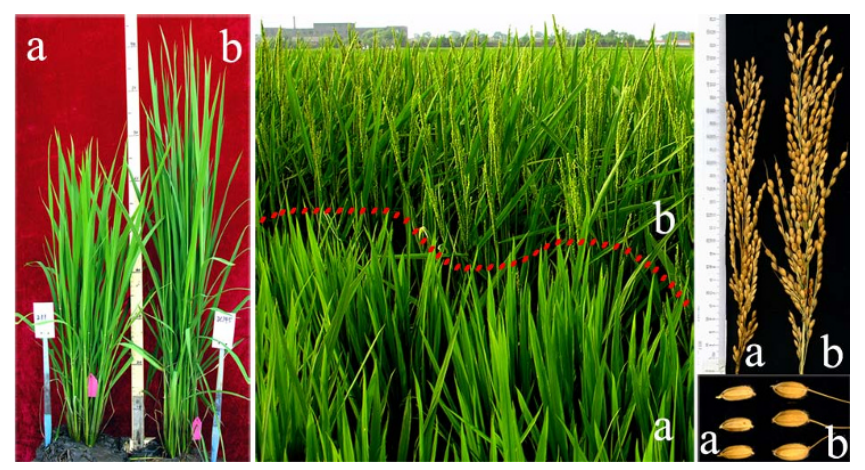

Figure I

Conspicuous phenotypic variation in multiple traits in selfed progenies (SI) of the single rice mutatorphenotype Tong2 I I-LP after its rice parental line cv. Tong2 I I was artificially pollinated by pollens of Oenothera biennis L. Leftmost and middle panels are overall plant statue of the rice parental line Tong2 I I (a) and SI progenies of the mutator-phenotype Tong2 I I-LP (b) at the vegetative and flowering stages, respectively. The rightmost panel exemplifies the panicle- and seed-sizes of the rice parental line Tong2 I I (a) and one individual of the SI progeny of Tong2 I I-LP (b). the question if some normally quiescent transposable elements (TEs) might have been activated in the mutatorphenotype, as in the case of Drosophila [30,31], and in bona fide wide hybrids of plants [32]. We thus checked stability of several previously characterized low-copy TEs endogenous to the rice genome, which were either documented to be active under certain stress conditions [3336], or were suspected so based on bioinformatic predictions [37]. The studied TEs included a 430 bp MITE, mPing [34], its two transposase donors, Ping and Pong [34,38], nine low-copy LTR retrotransposons, Osr2, Osr3, Osr7, Osr23, Osr35, Osr36, Osr42, Tos19 and Tos17 $[33,37]$. We found that four (mPing, Osr7, Tos17 and Osr23) of the 12 studied TEs showed apparent transpositional activation in Tong211-LP, or more often, in its S1 progenies (Figures 2, 3 and 4). A notable feature of the gel blotting patterns of these four TEs was that some of the S1 progenies exhibited individual-specific patterns, i.e., the pattern of a given individual was not shared by other sibindividuals, thus suggesting stochastic, transgenerational inheritance of the destabilized state in these elements, which mirrors the observation of phenotypic novelty in these plants Among the eight studied S1 individuals, \#5 showed the most transpositions for all four elements (Figures 2, 3 and 4). It remained to be determined if this "transpositional active state" would be continually inherited to future generations, or it would be converted to a stabilized, repressive state after a few generations.

Two (mPing and Tos17) of these four elements were shown previously as active under various stressful conditions, including tissue culture [34,35] and irradiation [36], but the other two (Osr7 and Osr23) were only implicated as potentially active based on bioinformatic predictions but not empirically documented [37]. Thus, this is the first demonstration of transpositional activity of these two LTR retrotransposons in rice under this specific condition (Figures $4 \mathrm{a}$ and 4c).

Next, we studied the transposition of mPing in Tong211LP and its S1 progenies in more detail, as this element is most active as well as amenable to characterization. First, the transpositional activity of mPing in these plants was further verified by transposon-display or TD [39], whereby $>60$ putative mPing excision and de novo insertion events were isolated and sequenced. The sequence data indicated that at least 52 of the isolated events represent bona fide mPing activities (i.e., excisions or de novo insertions; see Additional files 1 and 2), as they each contained at their 5' terminus the expected portion of the mPing sequence encompassing the typical 15 bp terminal inverted repeats (TIRs) and the 3-bp target site duplication (TSD) of TAA or TTA, which is characteristic of the transpositional behavior of mPing [34-36]. Based on the sequence information, together with the complete 


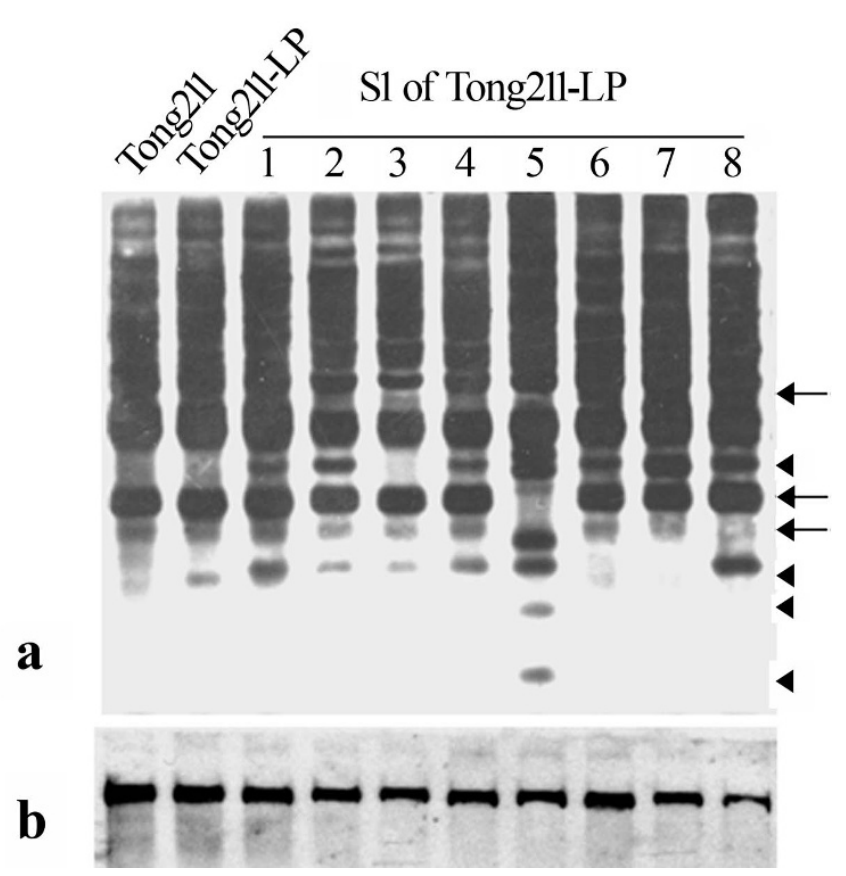

Figure 2

Transpostional activation of $m$ Ping revealed by DNAgel blotting. (a) Hybridization of the full-length of $m$ Ping to a blot containing Xbal-digested genomic DNA of the various lines, including the rice parental line Tong2 II, the mutatorphenotype Tong2 I I-LP and 8 individuals from its selfed progeny $(\mathrm{SI})$. Arrows and arrowheads denote positions of loss and gain of bands in the SI progenies of Tong2 I I-LP, respectively. (b) Hybridization of a Pong fragment in the ORF2 region (Pong-ORF2) to the same blot as in (a). The monomorphic single band in all lanes indicates that these rice plants likely contain a single copy of Pong, and which was transpositionally static.

genomic sequence of the standard laboratory rice genotype Nipponbare, locus-specific primers were designed for a set of 30 loci (see Additional files 1 and 2), which showed identical or high degree of sequence conservation between the studied rice cultivar (Tong211) and the genome-sequenced japonica rice cultivar Nipponbare. All these 30 loci were successfully amplified, cloned, sequenced and characterized (see Additional files 1 and 2). Sixteen of the 30 loci represent excisions in the S1 progenies of Tong211-LP, that is, compared with the parent Tong211 and the mutator-phenotype Tong211-LP (S0), they were excised in one or more of the S1 progenies (see Additional file 1). The predominant occurrence of mPing excisions in the S1 rather than the S0 generation (also evident in the gel blotting pattern, Figure 2) suggests that the timing of the excision events should be during late vegetative development and/or early gametogenesis in the Tong211-LP (S0) plant, and being manifested in the next generation via germline inheritance. Pairwise sequence comparison showed that none of 16 excisions had left behind any excision footprint (see Additional file $1)$, which is in agreement with some $[38,40]$ but not all (e.g., $[26,27])$ previous reports on the excision properties of mPing. A BlastN analysis of these 16 excised mPing loci against the whole genome sequence of Nipponbare revealed an unexpected result in that eight of 16 loci were mapped to chromosome 3 and the rest to chromosomes 1, 2, 4, 11 and 12 (see Additional file 1), suggesting differential activity of the mPing copies with regard to their chromosomal locations.

Fourteen of the TD-identified loci were mPing de novo insertions in the S1 progenies of Tong211-LP, i.e., compared with the parent Tong211 and Tong211-LP (S0) they became larger-sized due to insertion of intact mPing copies in some of the S1 progenies (see Additional file 2). A Blast $N$ analysis of these insertion-targeted sequences against the whole genome sequence of Nipponbare showed that all insertions mapped to unique- or low-copy regions (see Additional file 2), consistent with insertional propensity of $m$ Ping [41]. In contrast to the situation of excisions, these $14 \mathrm{mPing}$ insertions did not show an obvious bias towards a particular chromosome (see Additional file 2).

\section{Genome-wide genetic and epigenetic instability in the mutator-phenotype}

To test whether the genetic variations in Tong211-LP and its progenies were confined to the activation of a few specific TEs, or a more general genomic instabilities have been elicited, we performed genome-wide analysis on the same set of plants used in the gel-blotting by using AFLP and MSAP markers, and assessed $>1,000$ loci for each marker. In the AFLP analysis, both loss of the rice parental bands and gain of novel bands were detected in Tong211LP and its S1 progenies relative to their rice parent Tong211, with variation frequencies of both types of genomic changes together ranged from $2.66 \%$ to $8.16 \%$ (Figure 5). In MSAP analysis, methylation alteration of both CG and CNG at the CCGG sites (a prominent site for methylation modification in eukaryotes) were also detected in Tong211-LP and its S1 progenies relative to their rice parent Tong211, with frequencies of both types of DNA methylation alterations together ranged from $21.44 \%$ to $27.30 \%$ (Figure 5 ), which are higher by more than three times than those of genetic changes. These data indicated that, apart from transpositional activation of a subset of TEs (Figures 2, 3 and 4), pollination by pollens of $O$. biennis had particularly induced genome-wide epigenetic instabilities in the form of altered cytosine methylation patterns in the mutator-phenotype and its S1 progenies, though lower frequencies of genetic changes also occurred. It is notable that, as in the case for the trans- 


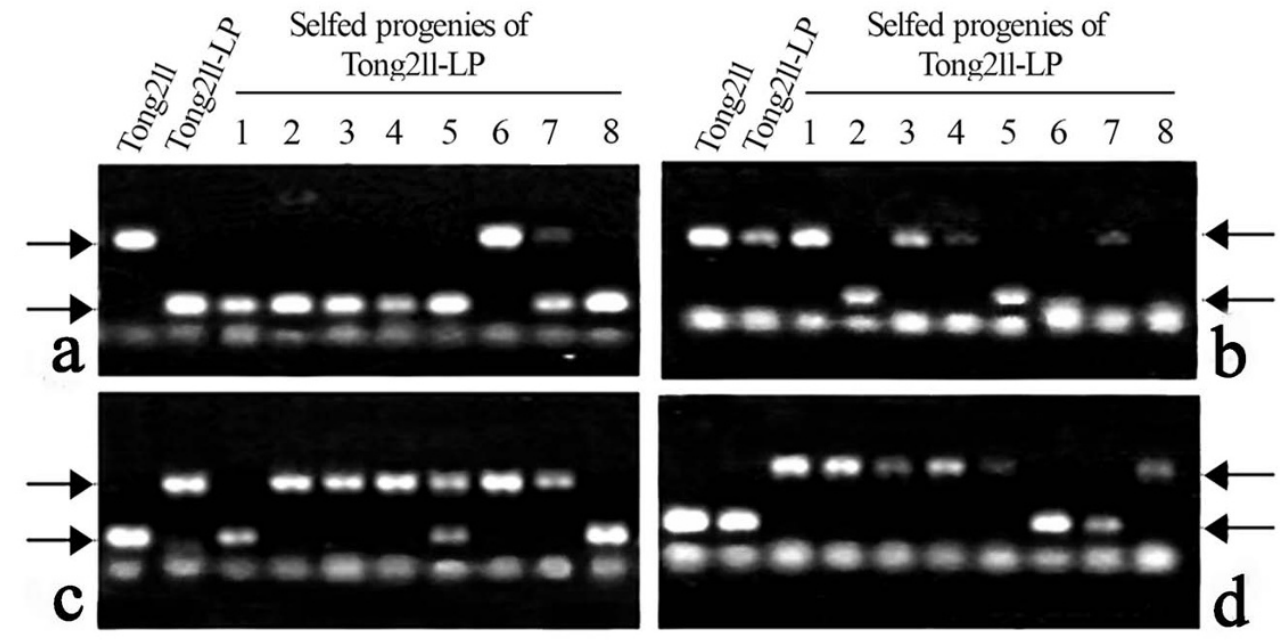

\section{Figure 3}

Examples of validation of $m$ Ping excision and insertion events by locus-specific PCR amplifications. PCR amplification products by using mPing-bracketing locus-specific primers on template DNA of the rice parent (Tong $2 \mathrm{I} I$ ), the mutant (Tong2 I I-LP) and its $8 \mathrm{SI}$ progeny individual. (a) and (b) are excisions of $m$ Ping from the mutant Tong2 I I-LP and/or some of its SI progenies, while (c) and (d) are de novo insertions in the mutant Tong2 I I-LP and/or some of its SI progenies. Arrows refer to positions of the upper larger-sized bands and lower smaller-sized bands, the size difference between which is exactly 430 bp (the full-length of $\mathrm{mPing}$ ), based on sequencing.

position of the four TEs, described above, many loss or gain of bands in the AFLP or MSAP profiles were singletons (a specific change occurred in only one individual), thus probably could not be attributed to genetic segregation from existing variations in the So generation alone. Instead, stochastic transgenerational inheritance of the induced metastable epigenetic chromatin state might be a major underlying cause for the new variations in the S1 progeny, which is apparently consistent with the de novo appearance of phenotypic variations in the S1 plants (e.g., Figure 1).

To gain some insights into the chromosomal distribution and possible functional relevance of the genomic loci that showed genetic and epigenetic instabilities in Tong211-LP and its progenies, a set of variable AFLP and MSAP bands were isolated, cloned and sequenced (see Additional file 3 ). It was found that all variable bands were chromosomal DNA sequences of the rice genome (thus again pointing to the lack of genetic introgression from the pollen donor $O$. biennis), and they mapped to all 12 rice chromosomes, with the numbers ranged from two to six for each chromosome. A notable observation from the inferred possible functionalities of these variable bands is that the majority of them (23 out of 41) appeared to be genic sequences, followed by TEs (11), and only seven showed no homology (see Additional file 3 ). This may explain the dramatic phenotypic variations in the mutator-phenotype and its selfed S1 progenies (e.g., Figure 1).

\section{Correlations between two of the three kinds of} instabilities, genetic variation, epigenetic variation, and TE activity

Given the concomitant occurrence of genetic variation, alteration in cytosine methylation and TE activity in the mutator-phenotype and its selfed progenies, it would be interesting to know whether these events are intrinsically correlated with, or independent of, each other. Thus, we calculated the Pearson's coefficients between each two of the three kinds of instabilities. We found no meaningful correlation between the genetic variation detected by AFLP and the alteration in cytosine methylation detected by MSAP (Table 1), suggesting the two kinds of variations were likely caused by independent mechanisms [42]. Likewisely, no correlation was seen between the genetic variation and mPing activity (including excision and insertion, detected by transposon-display) (Table 2), which is as expected given the known distinct cellular mechanisms for maintaining general genetic stability and control of TE activity. In contrast, statistically meaningful correlations were found between mPing activity and alteration in cytosine methylation (Table 2). Specifically, mPing excision is positively correlated with alteration in both CG and CNG methylation $(\mathrm{P}<0.05$ or 0.01$)$, and mPing insertion is positively correlated with alteration in CNG methylation $(\mathrm{P}<0.05)$ (Table 2). This is consistent with the genomedefense paradigm of cytosine methylation $[43,44]$. According to this paradigm, the primary role of cytosine methylation was evolved to serve as a genome defense sys- 


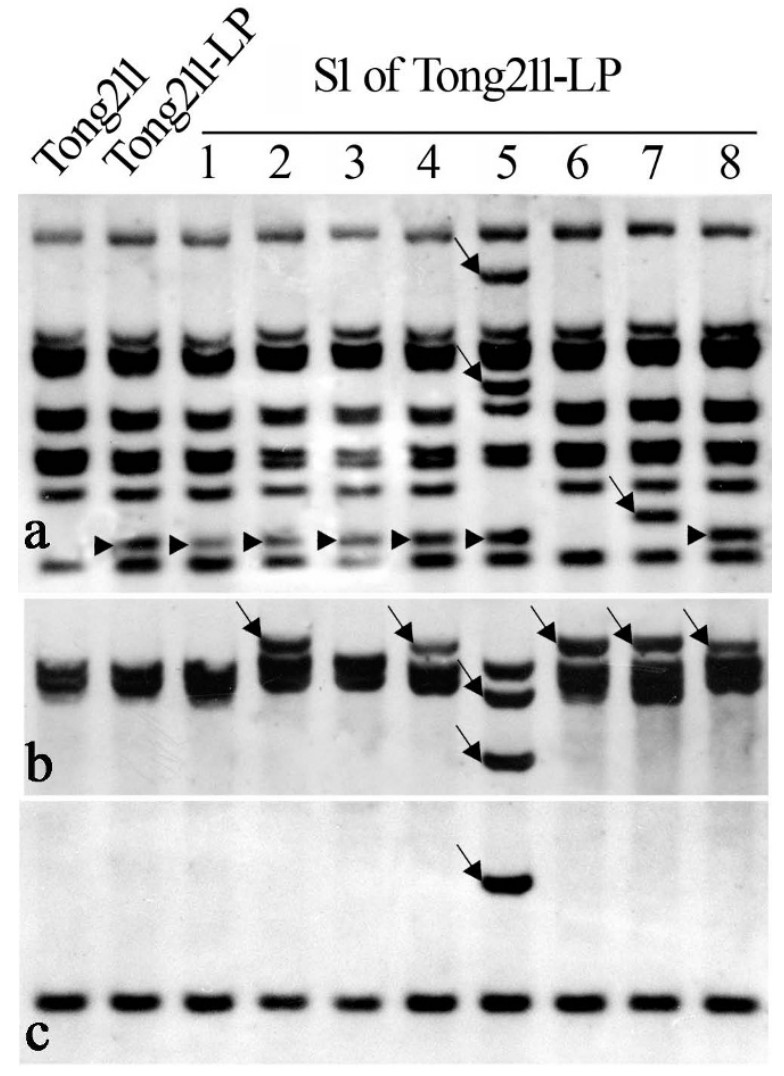

Figure 4

Transpositional mobilization of three LTR-retrotransposons revealed by DNA-gel blotting. (a), (b) and (c) are hybridization patterns for Osr7, Tos I 7 and Osr23, respectively. For each element, a portion of the reverse transcriptase (RT) region was amplified by specific primers (listed in Additional file 4) and used as a probe against the same blot as used in Figure 2. Arrows indicate novel bands appeared in the mutant Tong2 I I-LP or its SI progenies relative to their rice parent Tong2II, which are suggestive of de novo retrotranspositional events. Note that progeny \#5 showed the most rampant retrotranspositions, as is also the case for mPing (Figure 2); in addition, loss of bands was evident for two retrotransposons (Osr7 and Tos / 7) in this individual, suggesting the occurrence of genomic rearrangements within or adjacent to each of the element copies.

tem particularly to control mobility of endogenous TEs, and therefore stress-induced alteration in scope and/or extent of this epigenetic marker may provoke activation of otherwise quiescent TEs [43].

Heritable alteration in expression state of genes encoding for putative DNA methyltransferase, 5-methylcytosine DNA glycosylase and siRNA pathway-related protein in the mutator-phenotype and its progenies

Given that two known mechanisms, cytosine methylation and small interfering (si) RNA, often play critical roles in repressive control of TE activity [43-47], and the above documented correlation between alteration in cytosine methylation and TE (mPing) activity in the rice mutatorphenotype, it is reasonable to assume that the TE activity and epigenetic instability are probably related to perturbation of the homeostatic expression state of genes encoding for the enzymatic machinery responsible for maintaining the cytosine methylation states and/or other aspects of the chromatin epigenetic structure. We thus measured the mRNA steady-state abundance for a set of genes encoding for putative DNA methyltransferases, 5-methylcytosine DNA glycosylases, and the siRNA pathway-related proteins by real-time reverse transcriptase- PCR (q-RT-PCR) analysis with gene-specific primers. We found that of the 13 selected genes analyzed, 11 (the two exceptions are DDM1 and AGO4-2) showed significantly perturbed expressions in the mutator-phenotype (Tong211-LP) relative to its rice parental line (Tong211) (Figure 6). Furthermore, the perturbed expression states of these genes were transgenerationally heritable in the sense that none of the
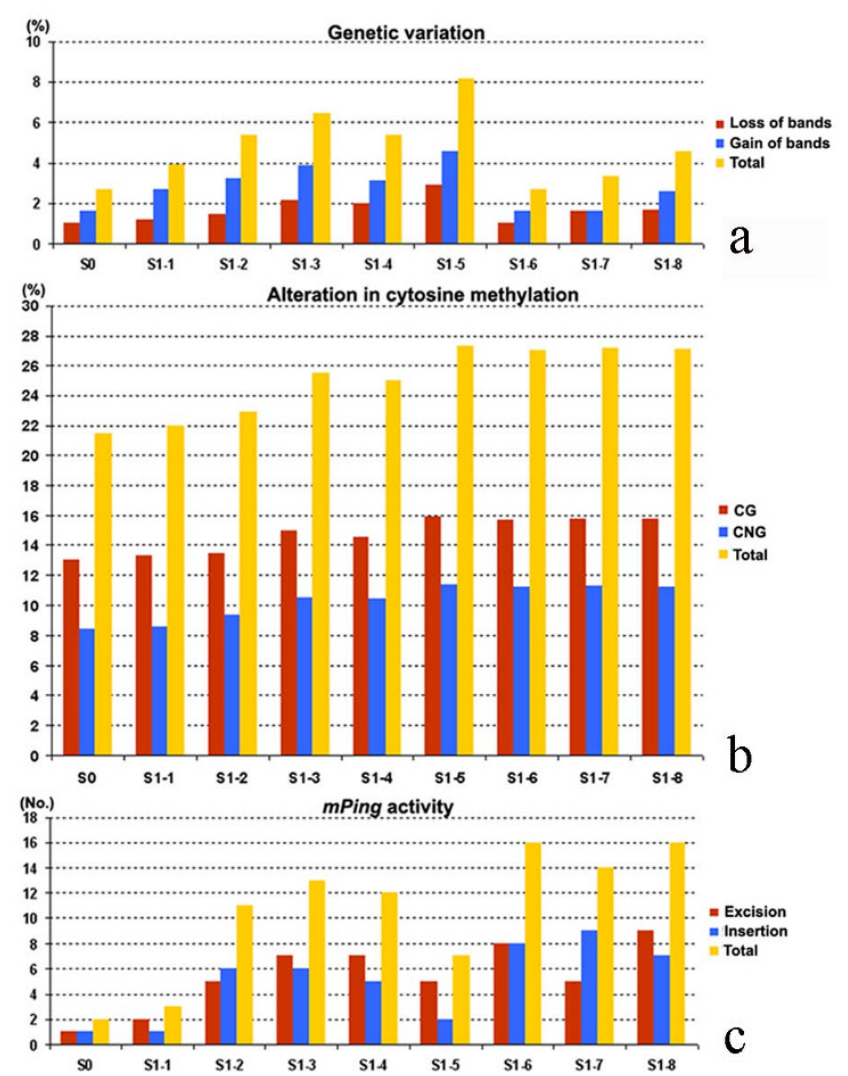

Figure 5

Summary of the frequency or number of genetic changes detected by AFLP (a), alterations in cytosine methylation (at the CCGG sites) detected by MSAP (b) and $m$ Ping transpostional activity revealed by transposon-display (TD) (c), in the mutator-phenotype Tong I I (S0) and its 8 SI progeny individuals. 
genes had reverted to the original expression states of their parental rice line (wild type) in all or most of the eight S1 plants analyzed (Figure 6). In fact, the extent of perturbation in the expression states of most of the genes was further augmented in some of the S1 progenies, and in one extreme case (i.e., the DDM1 gene), whose expression did not exhibit significant deviation from the parental line in Tong211-LP, nonetheless showed significant difference in six (except for S1-1 and -3) of the eight S1 individuals (Figure 6). This result of transgenerational perturbation in the expression states of the chromatin structure-maintenance genes is consistent with the heritable epigenetic instability in these plants, described above.

Recent studies have established that the intrinsic DNA methylation patterns in both plants and animals are faithfully maintained and perpetuated by coordinated function of at least two classes of DNA methyltransferases (maintenance and de novo), together with active demethylases, i.e., the 5-methylcytosine DNA glycosylases [48,49]. On the other hand, small interference RNA (siRNA) was documented as playing pivotal roles in repressing activity of TEs in diverse organisms by specific targeting [44-47]. Furthermore, at least in plants de novo methylation is often related to the activity of certain species of siRNAs by a mechanism known as RNA-directed DNA methylation or RdDM [50]. It is therefore conceivable that the coordinated expression of these genes represent a default requirement for stable maintenance and perpetuation of intrinsic DNA methylation patterns and silent TE states. Thus, the transgenerational perturbation of these genes in the mutator-phenotype and its progenies (Figure 6) may conceivably disrupt the homeostatic expression state of these genes as a network in the rice cells. It is likely that at least one facet of the possible effect of alien pollination as a "biological stress" may lie in its perturbation of coordinated expression of these chromatin state maintenance and regulation genes in the maternal recipient somatic and germinal cells, and hence, result in transgenerational epigenetic instability and TE activation.

\section{The mutator-phenotype was not caused by parental heterozygosity or contamination, but resultant from the alien pollination-imposed stress}

There are three alternative possible causes that may be responsible for or contribute to the generation of the mutator-phenotype: one is segregation of pre-existing parental heterozygosity, the second is seed or pollen contamination from other rice cultivar(s), and the third is mutagenic effect from some unknown source. That we consider pollination by $\mathrm{O}$. biennis as the only major underlying cause for the genetic and epigenetic instabilities in the mutator-phenotype (Tong211-LP) and its S1 progenies, are based on the following lines of evidence: First, the rice parental cultivar cv. Tong 211 is a genetically pure line, as rice is a predominantly self-pollinating plant, and furthermore, the specific strain used for the present work had been maintained by strict selfing for $>10$ successive generations in our hands, thus its inbred nature was ensured. In fact, the inbred nature of Tong211 has also been validated in this study by a parallel analysis on 30 random individuals, as in no case a variable pattern suggestive of heterozygosity was observed in either the gelblotting patterns of the four active TEs or in PCR-based locus-specific mPing amplifications of all 30 loci (see Additional files 1 and 2; data not shown). Therefore, parental heterozygosity can be confidently ruled out as a causal factor for the markedly changing patterns of either the studied TEs, and by extension, the variable MSAP/ AFLP profiles. Second, based on the following lines of evidence, contamination by pollens or seeds of other rice cultivars was considered as extremely unlikely. (1) Strict precautions were taken both in the cross manipulations (emasculation and pollination) and in later propagations by timely bagging of all panicles to endure $100 \%$ selfing. (2) It is notable that whereas most genetic and epigenetic changes that occurred in the mutator-phenotype were largely inheritable to its $\mathrm{S} 1$ progenies, many individualspecific new patterns appeared de novo in the S1 individuals (e.g., Figures 2, 3 and 4). Therefore, if contamination were a cause for the observed variable patterns, then the S1 plants need to have derived from S0 seeds that were contaminated independently by pollens of different rice cultivars, which obviously is extremely unlikely. (3) In the course of identifying the mPing-containing loci in cultivar Tong211, we uncovered 21 additional loci each contains a mPing copy in the standard cultivar Nipponbare but devoid the element in Tong211 (see Methods). We then amplified these 21 mPing-empty loci from the mutatorphenotype and its eight S1 progeny individuals, and we found that in all cases, only smaller-sized PCR products consistent with lacking of mPing in these plants were

Table I: Correlation between the genetic variations detected by AFLP and alteration in cytosine methylation detected by MSAP based on Pearson's coefficients

\begin{tabular}{|c|c|c|}
\hline \multirow[t]{2}{*}{ Genetic variation detected by AFLP } & \multicolumn{2}{|c|}{ Alteration in cytosine methylation detected by MSAP } \\
\hline & CG & CNG \\
\hline $\begin{array}{l}\text { Loss } \\
\text { Gain }\end{array}$ & $\begin{array}{l}0.488 \\
\left(P_{0.05}=0.183\right) \\
0.123 \\
\left(P_{0.05}=0.753\right)\end{array}$ & $\begin{array}{l}0.515 \\
\left(P_{0.05}=0.156\right) \\
0.163 \\
\left(P_{0.05}=0.673\right)\end{array}$ \\
\hline
\end{tabular}


Table 2: Correlations of $m$ Ping activity respectively with the genetic variations detected by AFLP and alteration in cytosine methylation detected by MSAP, based on Pearson's coefficients

\begin{tabular}{|c|c|c|c|c|}
\hline \multirow[t]{2}{*}{ mPing activity detected by TD } & \multicolumn{2}{|c|}{ Genetic variation detected by AFLP } & \multicolumn{2}{|c|}{$\begin{array}{l}\text { Alteration in cytosine methylation } \\
\text { detected by MSAP }\end{array}$} \\
\hline & loss & gain & CG & CNG \\
\hline $\begin{array}{l}\text { excision } \\
\text { insertion }\end{array}$ & $\begin{array}{l}0.286 \\
\left(P_{0.05}=0.455\right) \\
-0.075 \\
\left(P_{0.05}=0.848\right)\end{array}$ & $\begin{array}{l}0.166 \\
\left(P_{0.05}=0.670\right) \\
-0.286 \\
\left(P_{0.05}=0.456\right)\end{array}$ & $\begin{array}{l}0.732^{*} \\
\left(P_{0.05}=0.025\right) \\
0.609 \\
\left(P_{0.05}=0.08 \mathrm{I}\right)\end{array}$ & $\begin{array}{l}0.800 * * \\
\left(P_{0.01}=0.010\right) \\
0.675^{*} \\
\left(P_{0.05}=0.046\right)\end{array}$ \\
\hline
\end{tabular}

$*$ and $* *$ respectively indicate statistical significance at the 0.05 and 0.01 levels.

amplified (data not shown). Given the extremely high degree of presence $v$ s. absence polymorphism of mPing among japonica rice cultivars [[34,51]; our unpublished data], this result strongly suggests that the mutator-phenotype and its analyzed progenies were unequivocally originated from one cultivar (Tong211) only. Taken together, the possibility of pollen or seed contamination can be confidently ruled out. Finally, all plant lines used in this study were grown together under identical normal conditions, under which biotic (e.g., pathogen infesta- tion) and abiotic stresses were not exerted. Therefore, it is also inconceivable that the mutator-phenotype and its progenies had been differentially stressed from their parental line by an unknown stress that elicited the genomic instabilities.

By ruling out each of the three alternative possibilities, we are confident to conclude that pollination by $O$. biennis is the major, if not the only, conceivable cause for the genetic and epigenetic variations in the mutator-pheno-
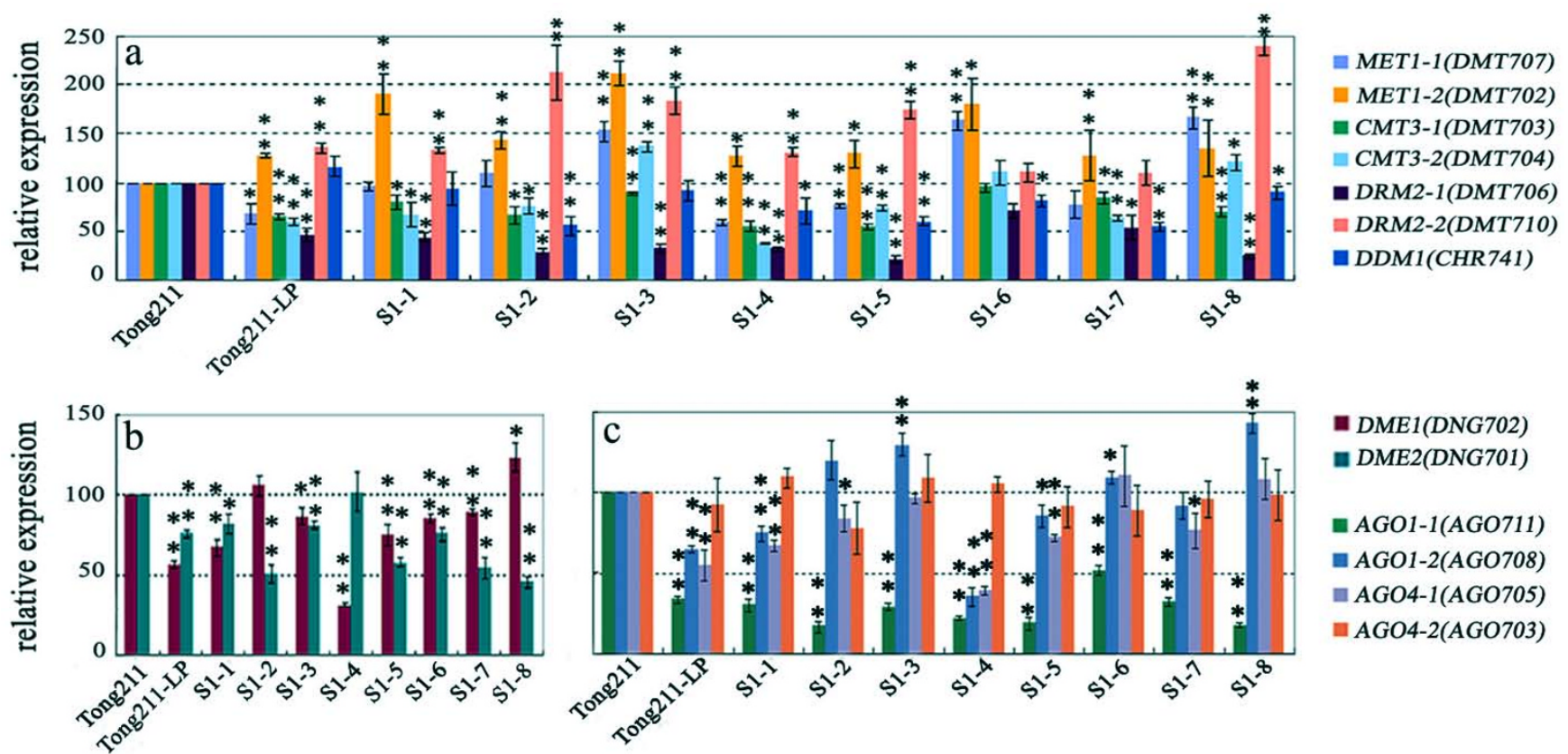

- DME1(DNG702) - DME2(DNG701)

- AGO1-1(AGO711)

- AGO1-2(AGO708)

- $A G 04-1$ (AGO705)

- AGO4-2(AGO703)

Figure 6

Alteration in transcript abundance of a set (13) of genes encoding for putative DNA methyltransferases (a), 5methylcytosine DNA glycosylases (b), and siRNA pathway-related proteins (c) in the mutator-phenotype (Tong2 I I-LP) and 8 selected SI individuals, relative to their parental rice line Tong2 I I. Real-time RT-PCR analysis on transcript quantity of the 13 genes in expanded young leaf tissue was performed on three batches of independent RNAderived cDNAs with gene - specific primers (see Additional file 5). The relative abundance of transcripts (means \pm SD) for each of the studied genes was calculated upon normalization against a rice $\beta$-actin gene (Genbank accession $\underline{X 79378)}$ ). The gene names are labeled. * and $* *$ denote statistical significance at the 0.05 and 0.01 levels, respectively. 
type and its sefled progenies. Nonetheless, the basis for the occurrence of such extensive genetic and epigenetic instabilities as a result of "abortive" alien pollination remains mysterious. The single mutator-phenotype (Tong211-LP) was identified out of 84 "pollinated" plants, based on its striking phenotypic variations, thus giving a mutation frequency of $1.2 \%$. Although it is likely that genomic variations may also have occurred in some other treated plants, they did not reach the extent to cause apparent phenotypic variations. Because all these 84 plants were sequentially pollinated first by $O$. biennis and then by pollens from the same rice line (Tong211), it appeared likely that stochasticity have also played an important part in the genesis of the mutator-phenotype individual.

The phenomenon we reported here is reminiscent of what McClintock envisioned two decades ago that wide hybridization in plants might activate quiescent TEs and cause genomic restructuring [18]. Indeed, several lines of empirical evidence in both plants and animals have lend support to this prediction $[8,13,30-32,40,52-55]$. Although all these previous works involved documented genome merger and/or introgression, it can be envisioned that even in the absence of introgression a "shock" at multiple levels may be incurred if the pollination by alien species per se represents a kind of "biological stress". In principle, even between incompatible crosses, certain metabolites, particularly those that require only trace quantity to produce dramatic effects like signal molecules, phytohormones and siRNA species etc., may be released from the donor pollens and enter the recipient stigma cells, thus may conceivably produce various mismatches and elicit a stress response.

It can be imagined that the cellular machinery responsible for safeguarding the genetic and epigenetic stabilities is likely sensitive as well as responsive to perturbations by stress, and fine-tuning on a balance between genetic/epigenetic fidelity and instability is required for the sake of survival and adaptation. Thus, in this study the significantly perturbed expression of nearly all of the 13 studied genes involved in the cellular machinery responsible for maintenance of chromatin epigenetic state subsequent to alien pollination might represent a sensory and adaptive response by the plant genome. From this perspective, the findings of this study may have bearing to genome evolution, as similar incidents of alien pollination may occur frequently under natural conditions, and hence, implicate a novel role of hybridization in evolution. Thus, we propose the possibility that "accidental cross-pollination" by a certain unrelated species may be actually mutagenic and elicited dramatic genetic/epigenetic instabilities, which may be perceived by selection. Further judiciously designed experiments involving an array of cross manipu- lations between different plant species are needed to investigate generality of this phenomenon and its underlying mechanism.

\section{Conclusion}

To test the possibility that pollination by an unrelated and incompatible species may constitutes a "biological stress" whereby the genetic and epigenetic stability of the maternal parent genome might be jeopardized, we performed a crossing experiment between rice (served as the maternal partner) and Oenothera biennis L. (served as the pollen donor). A single rice mutator-phenotype individual (Tong211-LP) with conspicuous variation in multiple phenotypic traits was identified from the crossing experiment. Tong211-LP and its sefled progenies exhibited transgenerational epigenetic instability in the form of altered cytosine methylation and transpositional activation of several otherwisely quiescent transposable elements (TEs) endogenous to the rice genome. Heritably perturbed homeostatic expression-state of a set of genes involved in maintenance of chromatin structure is likely an underlying cause for the alien pollination-induced transgenerational epigenetic/genetic instability, and which occurred apparently without entailing genome merger or genetic introgression. Our results suggest that accidental pollination by unrelated alien pollens in plants might impose a stress condition and induce genetic and epigenetic instabilities in the maternal genome.

\section{Methods}

\section{Plant materials}

Plants used in this study included a single rice individual named "Tong211-LP" that was identified from a set of plants derived from seeds of a "alien pollination experiment" between rice (Oryza sativa L.), ssp. japonica, cv. Tong211 and a dicot plant, evening primrose (Oenothera biennis L.), and followed by self-pollination with pollens of different individuals of the same rice cultivar, namely, using a procedure we termed "repeated pollination" $[27,40]$. The choice for the particular crossing partners was based on two major considerations: (1) the two species (O. sativa L. and O. biennis L.) are hardly related, and hence, served well for the purpose of this study; (2) O. biennis L. produces a large amount of pollens that are viable for relatively long period after collection (our unpublished observation), and hence, convenient for the crossing manipulations. The identified individual plant (Tong211-LP) exhibited conspicuous phenotypic variation in multiple traits particularly enlarged panicles and seed-size, compared with its maternal parental cultivar Tong211. Seeds of individual panicles collected from Tong211-LP were selfed to produce the S1 progenies. In all cases, mutant plants were grown together with the parental line under identical, normal conditions, and strict bagging was practiced. 


\section{DNA gel blot analysis}

Genomic DNA was isolated from expanded young leaves of individual plants by a modified CTAB method [56] and purified by phenol extractions. Genomic DNA ( $3 \mu \mathrm{g}$ per lane) was digested by XbaI (New England Biolabs Inc.), and run through $1 \%$ agarose gels. The choice of $\mathrm{XbaI}$ is because the studied TEs either do not have a restriction site or the site(s) being on one side of the probe region, such that copy number of the TEs can be estimated based on the blotting patterns. Fractionated DNA was transferred onto Hybond $\mathrm{N}+$ nylon membranes (Amersham Pharmacia Biotech) by the alkaline transfer recommended by the supplier. For investigating stability of a set of 13 low-copy transposable elements (TEs), element-specific primers were designed (see Additional file 4), and the fragments were obtained by PCR amplifications by using genomic DNA of the parental line (Tong211) as the template. The fragments representing each of the TEs were then gel-purified, identities confirmed by sequencing, and labeled with fluorescein-11-dUTP by the Gene Images random prime-labeling module (Amersham Pharmacia Biotech). Hybridization signal was detected by the Gene Images CDP-Star detection module (Amersham Pharmacia Biotech) after washing at a stringency of $0.2 \times$ SSC, $0.1 \%$ SDS for $2 \times 50 \mathrm{~min}$. The filters were exposed to X-ray film for 1-3 hrs depending on signal intensity.

\section{Transposon-display (TD) and PCR-based locus assay on mPing excision and insertion}

The transposon-display (TD) technique [39], using nested mPing-specific primers together with a primer designed according to the restriction enzyme MseI-adapter sequence was as described [40]. To further verify mPing excisions and insertions, a subset of identified TD loci were sequenced, and by taking advantage of the complete genome sequence of the standard laboratory japonica rice cultivar Nipponbare http://rgp.dna.affrc.go.jp, a set of locus-specific primers (see Additional file 1) each bracketing an intact mPing in the parental rice cultivar Tong211 (for detecting excision) or in the mutant Tong211-LP and its S1 progeny individual(s) (for detecting insertions), was designed by the Primer 3 software http://biocore.unl.edu/ cgi-bin/primer3/primer3 www.cgi. Likewisely, a set of 21 loci each of which does not encompass a mPing copy in the parental cultivar Tong-211 was also identified in the course of TD analysis. Primers specific to this set of loci were also designed, and used to validate single genotypic origin of the mutant (Tong-211-LP) and its progenies. PCR amplification with these primer pairs were then conducted on the corresponding plant materials. The amplicons were visualized by ethidium bromide staining after electrophoresis through $2 \%$ agarose gels. All identified sites for mPing excisions (along with the corresponding element-containing donor sites) and de novo insertions were isolated and sequenced, such that the excision pros- perities (e.g., to leave footprint or not) and characteristics (e.g., chromosomal location and potential functionality) of insertion-targeted sequences could be determined or inferred.

\section{AFLP and MSAP analysis}

The protocols suitable for amplified fragment length polymorphism (AFLP) and methylation-sensitive amplified fragment (MSAP) in rice were exactly as reported [22,57]. For each marker, $>1,000$ loci were scored. Typical bands representing genetic changes (AFLP) and cytosine methylation alterations (MSAP) in the mutant or its S1 progenies, as compared with their parental cultivar Tong211, were isolated, cloned and sequenced. Homology analysis was performed by BlastX at the NCBI website http:// www.ncbi.nlm.nih.gov/blast/Blast.cgi.

\section{Real-time Reverse transcriptase (RT)-PCR analysis}

Isolation of total RNA and CDNA synthesis was essentially as reported [24]. Specifically, total RNA was isolated from expanded young leaves at the same developmental stage as that used for DNA isolation by the Trizol Reagent (Invitrogen), following the manufacturer's protocol. The RNA was treated with DNaseI (Invitrogen), reverse-transcribed by the SuperScriptTM RNase H-Reverse Transcriptase (Invitrogen), and subjected to q-RT-PCR analysis using gene-specific primers. The q-PCR experiments were performed using a Roche LightCycler480 apparatus (Roche Inc.) according to the manufacturer's instruction and SYBR Premix Ex Taq (Takara) as a DNA-specific fluorescent dye. The primers for all 13 studied genes encoding for putative DNA methyltransferases (seven), 5-methylcytosine DNA glycosylases (two) and siRNA-related proteins (four) were designed by the Primer 5 software (see Additional file 5). Expression of a rice $\beta$-actin gene (Genbank accession $\underline{\mathrm{X} 79378}$ ) was used as internal control with the primer pairs 5'-ATGCCATTCTCCGTCTT-3' and 5'-GCTCCTGCTCGTAGTC-3'. Thermal cycling conditions consisted of an initial denaturation step at $95^{\circ} \mathrm{C}$ for $30 \mathrm{~s}$, followed by 45 cycles of $15 \mathrm{~s}$ at $95^{\circ} \mathrm{C}$ and $1 \mathrm{~min}$ at $60^{\circ} \mathrm{C}$. Three batches of independently isolated RNAs were used as technical replications. The melting curve analysis with the LightCycler480 together with 1.5\% agarose gel electrophoresis of the products were used to ensure that right size product without significant background was amplified in the reaction. The relative amounts of the gene transcripts were determined using the Ct (threshold cycle) method, as described by the manufacturer's protocol. Data were analyzed by using the software provided by Roche Company and calculated by the $2-\Delta \Delta \mathrm{Ct}$ method. Quantitative results were given as mean expression (means $\pm \mathrm{SD}$ ).

\section{Statistics}

Statistical significance was determined using SPSS 11.5 for Windows http://www.spss.com/statistics/ and analyzed 
by Independent-Samples student's t-Test. Specifically, correlations were tested for the data of variation frequencies calculated based on the three markers, Transposon (mPing)-display, MSAP and AFLP, by using the Pearson correlation analysis. The same program was used to test for the statistical significance of differences in the relative expression of the set of 13 genes in the mutator-phenotype (Tong211-LP) and its sefled progenies relative to that of the rice parental line (Tong211).

\section{Authors' contributions}

HYW, YC, YYZ, YW, and FN carried out the laboratory experiments, analyzed the data and participated in drafting the manuscript. XCC and JHZ performed all pollination work and maintained plants. CMX and BL designed the study and finalized the manuscript. All authors read and approved the final manuscript.

\section{Additional material}

\section{Additional file 1}

Characterization of $\mathrm{mPing}$ excisions. A total of 16 loci that were excised from one or more of the 8 studied progeny individuals (from $S_{1}-1$ to $S_{1}-$ 8) of the mutator-phenotype Tong211-LP $\left(S_{0}\right)$ were identified by mPingspecific transposon-display (TD) and validated by cloning, sequencing, and locus-specific PCR amplification.

Click here for file

[http://www.biomedcentral.com/content/supplementary/14712229-9-63-S1.doc]

\section{Additional file 2}

Characterization of $\mathrm{mPing}$ de novo insertions. A total of $14 \mathrm{mPing}$ de novo insertion events which occurred in some of the selfed progeny individuals (from $S_{1}-1$ to $S_{1}-8$ ) of the mutator-phenotype Tong211$L P\left(S_{0}\right)$ were identified by mPing-specific transposons-display (TD) and validated by cloning, sequencing and locus-specific PCR amplification. Click here for file

[http://www.biomedcentral.com/content/supplementary/14712229-9-63-S2.doc]

\section{Additional file 3}

Characterization of variant AFLP and MSAP bands. Chromosomal location and functional homology of isolated variant AFLP and MSAP fragments from the mutator phenotype Tong211-LP $\left(S_{0}\right)$ and/or its 8 selfed progeny individuals (from $S_{1}-1$ to $S_{1}-8$ ) were determined based on the reference genome sequence of $c v$. Nipponbare.

Click here for file

[http://www.biomedcentral.com/content/supplementary/14712229-9-63-S3.doc]

\section{Additional file 4}

Primers used to amplify the probe fragments of 12 low-copy and potentially active transposable elements (TEs) endogenous to the rice genome. Authenticity of the amplicons were verified by sequencing. Click here for file

[http://www.biomedcentral.com/content/supplementary/14712229-9-63-S4.doc]

\section{Additional file 5}

Real-time q-RT PCR primers used to measure the transcript quantity of a set of genes related to the chromatin epigenetic maintenance machinery. Primers of a set of 13 genes encoding for the putative DNA methyltransferases, 5-methylcytosine DNA glycosylases and siRNArelated proteins were designed by the Primer 5 software, based on the sequence information deposited at the Chromatin database http:// www.chromdb.org/.

Click here for file

[http://www.biomedcentral.com/content/supplementary/14712229-9-63-S5.doc]

\section{Acknowledgements}

This study was supported by the State Key Basic Research and Development Plan of China (2005CB I 20805), the Program for Changjiang Scholars and Innovative Research Team (PCSIRT) in University (\#IRT05 I9), and the National Natural Science Foundation of China.

\section{References}

I. Wendel JF: Genome evolution in polyploids. Plant Molecular Biology 2000, 42(I):225-249.

2. Rieseberg LH, Widmer A, Arntz AM, Burke JM: The genetic architecture necessary for transgressive segregation is common in both natural and domesticated populations. Philos Trans $R$ Soc Lond B Biol Sci 2003, 358( I 434): I I 4 |- I I 47.

3. Adams KL: Evolution of duplicate gene expression in polyploid and hybrid plants. Journal of Heredity 2007, 98(2):|36-|4|.

4. Stebbins GL: The role of hybridization in evolution. Proceedings of the American Philosophical Society 1959, 103:23 I-25I.

5. Grant V: Plant speciation. New York: Columbia; 1981.

6. Arnold ML: Natural Hybridization and Evolution. New York: Oxford University Press; 1997.

7. Matzke MA, Mittelsten Scheid O, Matzke AJ: Rapid structural and epigenetic changes in polyploid and aneuploid genomes. Bioessays 1999, 21 (9):761-767.

8. Osborn TC, Chris Pires J, Birchler JA, Auger DL, Chen Z], Lee HS, Comai L, Madlung A, Doerge RW, Colot V, Martienssen RA: Understanding mechanisms of novel gene expression in polyploids. Trends in Genetics 2003, 19(3): 141-147.

9. Doyle JJ, Doyle JL, Rauscher JT, Brown AHD: Diploid and polyploid reticulate evolution throughout the history of the perennial soybeans (Glycine subgenus Glycine). New Phytologist 2004, I6I(I): $121-132$.

10. Soltis DE, Soltis PS, Tate JA: Advances in the study of polyploidy since Plant speciation. New Phytologist 2004, I6I(1): I73-191.

II. Adams KL, Wendel JF: Novel patterns of gene expression in polyploid plants. Trends in Genetics 2005, 2 I (I 0):539-543.

12. Chen ZJ: Genetic and epigenetic mechanisms for gene expression and phenotypic variation in plant polyploids. Annual Review of Plant Biology 2007, 58:377-406.

13. Michalak P: Epigenetic, transposon and small RNA determinants of hybrid dysfunctions. Heredity 2009, I 02(I):45-50.

14. Michalak P, Noor MA: Genome-wide patterns of expression in Drosophila pure species and hybrid males. Mol Biol Evol 2003, 20(7): $1070-1076$.

15. Hegarty MJ, Barker GL, Wilson ID, Abbott RJ, Edwards KJ, Hiscock S): Transcriptome shock after interspecific hybridization in senecio is ameliorated by genome duplication. Curr Biol 2006, 16(16): 1652-1659.

16. Josefsson C, Dilkes B, Comai L: Parent-dependent loss of gene silencing during interspecies hybridization. Curr Biol 2006, 16(13): 1322-1328.

17. Doyle J], Flagel LE, Paterson AH, Rapp RA, Soltis DE, Soltis PS, Wendel JF: Evolutionary genetics of genome merger and doubling in plants. Annu Rev Genet 2008, 42:443-46I. 
18. McClintock B: The significance of responses of the genome to challenge. Science 1984, 226(4676):792-80 I.

19. Remus R, Kammer C, Heller H, Schmitz B, Schell G, Doerfler W: Insertion of foreign DNA into an established mammalian genome can alter the methylation of cellular DNA sequences. J Virol 1999, 73(2): 1010-1022

20. Heller H, Kammer C, Wilgenbus P, Doerfler W: Chromosomal insertion of foreign (adenovirus type 12, plasmid, or bacteriophage lambda) DNA is associated with enhanced methylation of cellular DNA segments. Proc Natl Acad Sci USA 1995, 92(I 2):55I5-55I9.

21. Liu Z, Wang Y, Shen Y, Guo W, Hao S, Liu B: Extensive alterations in DNA methylation and transcription in rice caused by introgression from Zizania latifolia. Plant Mol Biol 2004, 54(4):57|-582.

22. Wang $Y M$, Dong $Z Y$, Zhang ZJ, Lin $X Y$, Shen $Y$, Zhou D, Liu B: Extensive de Novo genomic variation in rice induced by introgression from wild rice (Zizania latifolia Griseb.). Genetics 2005, I 70(4): 1945-1956.

23. Jin H, Tan G, Brar DS, Tang M, Li G, Zhu L, He G: Molecular and cytogenetic characterization of an Oryza officinalis-O. sativa chromosome 4 addition line and its progenies. Plant Mol Bio 2006, 62(4-5):769-777.

24. Mclnnis SM, Desikan R, Hancock JT, Hiscock SJ: Production of reactive oxygen species and reactive nitrogen species by angiosperm stigmas and pollen: Potential signalling crosstalk? New Phytologist 2006, I72(2):22I-228.

25. Kovalchuk I, Kovalchuk O, Kalck V, Boyko V, Filkowski J, Heinlein M, Hohn B: Pathogen-induced systemic plant signal triggers DNA rearrangements. Nature 2003, 423(6941):760-762.

26. Boyko A, Kathiria P, Zemp F], Yao Y, Pogribny I, Kovalchuk I: Transgenerational changes in the genome stability and methylation in pathogen-infected plants: (virus-induced plant genome instability). Nucleic Acids Res 2007, 35(5): |7|4- 1725

27. Liu B, Piao HM, Zhao FS, Zhao JH, Zhao R: Production and molecular characterization of rice lines with introgressed traits from a wild species of Zizania latifolia Griseb. J Genet Breed 1999, 53(279-284):

28. Cooley L, Kelley R, Spradling A: Insertional mutagenesis of the Drosophila genome with single $\mathbf{P}$ elements. Science 1988, 239(4844): ||$|2|-|| 28$.

29. Liu B, Liu ZL, Li XW: Production of a highly asymmetric somatic hybrid between rice and Zizania latifolia (Griseb): Evidence for inter-genomic exchange. Theor Appl Genet 1999, 98:1099-II03.

30. Petrov DA, Schutzman JL, Hartl DL, Lozovskaya ER: Diverse transposable elements are mobilized in hybrid dysgenesis in Drosophila virilis. Proc Natl Acad Sci USA 1995, 92(I 7):8050-8054.

31. Labrador M, Farre M, Utzet F, Fontdevila A: Interspecific hybridization increases transposition rates of Osvaldo. Mol Biol Evol 1999, I6(7):931-937.

32. Ungerer MC, Strakosh SC, Zhen Y: Genome expansion in three hybrid sunflower species is associated with retrotransposon proliferation. Curr Biol 2006, I6(20):R872-873.

33. Hirochika $H$, Sugimoto K, Otsuki $Y$, Tsugawa H, Kanda M: Retrotransposons of rice involved in mutations induced by tissue culture. Proc Natl Acad Sci USA 1996, 93(15):7783-7788.

34. Jiang N, Bao Z, Zhang X, Hirochika H, Eddy SR, McCouch SR, Wessler SR: An active DNA transposon family in rice. Nature 2003, 42I(6919):163-167.

35. Kikuchi K, Terauchi K, Wada M, Hirano HY: The plant MITE mPing is mobilized in anther culture. Nature 2003, 42 I(6919): $167-170$

36. Nakazaki T, Okumoto Y, Horibata A, Yamahira S, Teraishi M, Nishida $\mathrm{H}$, Inoue $\mathrm{H}$, Tanisaka $\mathrm{T}$ : Mobilization of a transposon in the rice genome. Nature 2003, 42I(69 I9): 170-172

37. Gao L, McCarthy EM, Ganko EW, McDonald JF: Evolutionary history of Oryza sativa LTR retrotransposons: a preliminary survey of the rice genome sequences. BMC Genomics 2004, 5(I): 18.

38. Yang G, Zhang F, Hancock CN, Wessler SR: Transposition of the rice miniature inverted repeat transposable element $\mathrm{mPing}$ in Arabidopsis thaliana. Proc Natl Acad Sci USA 2007, 104(26): 10962-10967.

39. Broeck D Van den, Maes T, Sauer M, Zethof J, De Keukeleire $P$, D'Hauw M, Van Montagu M, Gerats T: Transposon Display iden- tifies individual transposable elements in high copy number lines. Plant J 1998, I3(1): I2I-129.

40. Shan X, Liu Z, Dong Z, Wang Y, Chen Y, Lin X, Long L, Han F, Dong $Y$, Liu B: Mobilization of the active MITE transposons mPing and Pong in rice by introgression from wild rice (Zizania latifolia Griseb.). Mol Biol Evol 2005, 22(4):976-990.

4I. Naito K, Cho E, Yang G, Campbell MA, Yano K, Okumoto Y, Tanisaka $T$, Wessler SR: Dramatic amplification of a rice transposable element during recent domestication. Proc Natl Acad Sci USA 2006, I 03(47): I 7620-I7625

42. Richards E): Inherited epigenetic variation - revisiting soft inheritance. Nat Rev Genet 2006, 7(5):395-40I.

43. Yoder JA, Walsh CP, Bestor TH: Cytosine methylation and the ecology of intragenomic parasites. Trends Genet 1997, I3(8):335-340.

44. O'Donnell KA, Boeke JD: Mighty Piwis defend the germline against genome intruders. Cell 2007, I29(I):37-44.

45. Jensen S, Gassama MP, Heidmann T: Taming of transposable elements by homology-dependent gene silencing. Nat Genet 1999, 2 | (2):209-2|2.

46. Sijen T, Plasterk RH: Transposon silencing in the Caenorhabditis elegans germ line by natural RNAi. Nature 2003, 426(6964):3|0-3|4.

47. Mallory AC, Vaucheret $\mathrm{H}$ : MicroRNAs: something important between the genes. Curr Opin Plant Biol 2004, 7(2): I20-I 25.

48. Morales-Ruiz T, Ortega-Galisteo AP, Ponferrada-Marin MI, MartinezMacias MI, Ariza RR, Roldan-Arjona T: DEMETER and REPRESSOR OF SILENCING I encode 5-methylcytosine DNA glycosylases. Proc Natl Acad Sci USA 2006, I 03(18):6853-6858.

49. Boyko A, Kovalchuk I: Epigenetic control of plant stress response. Environ Mol Mutagen 2008, 49(I):6I-72.

50. Matzke M, Kanno T, Huettel B, Daxinger L, Matzke AJ: Targets of RNA-directed DNA methylation. Curr Opin Plant Biol 2007, I0(5):5|2-5I9.

51. Huang X, Lu G, Zhao Q, Liu X, Han B: Genome-wide analysis of transposon insertion polymorphisms reveals intraspecific variation in cultivated rice. Plant Physiol 2008, I 48( I):25-40.

52. Capy P, Chakrani F, Lemeunier F, Hartl DL, David JR: Active mariner transposable elements are widespread in natural populations of Drosophila simulans. Proc Biol Sci 1990 242(1303):57-60.

53. Kidwell MG, Lisch DR: Hybrid genetics. Transposons unbound. Nature 1998, 393(6680):22-23.

54. O'Neill RJ, O'Neill MJ, Graves JA: Undermethylation associated with retroelement activation and chromosome remodelling in an interspecific mammalian hybrid. Nature 1998, 393(6680):68-72.

55. Liu B, Wendel JF: Retrotransposon activation followed by rapid repression in introgressed rice plants. Genome 2000, 43(5):874-880.

56. Kidwell KK, Osborn TC: Simple plant DNA isolation procedures. In Plant genomes: Methods for Genetic and Physical Mapping Kluwer Academic Publishers; 1992: I- 13.

57. Dong ZY, Wang YM, Zhang ZJ, Shen Y, Lin XY, Ou XF, Han FP, Liu $B$ : Extent and pattern of DNA methylation alteration in rice lines derived from introgressive hybridization of rice and Zizania latifolia Griseb. Theor Appl Genet 2006, I I3(2): 196-205. 